\title{
Pulmonary hypertension adversely affects short- and long-term survival after mitral valve operation for mitral regurgitation: Implications for timing of surgery
}

\author{
Mehrdad Ghoreishi, MD, ${ }^{a}$ Charles F. Evans, MD, ${ }^{a}$ Christopher R. DeFilippi, MD, ${ }^{b}$ Gerald Hobbs, PhD, ${ }^{c}$ \\ Cindi A. Young, ${ }^{a}$ Bartley P. Griffith, MD, ${ }^{a}$ and James S. Gammie, MD $^{\mathrm{a}}$
}

\begin{abstract}
Objective: We investigated the impact of preoperative pulmonary hypertension $(\mathrm{PH})$ on early and late outcomes after mitral valve operation for mitral regurgitation.

Methods: Systolic pulmonary artery pressure (sPAP) was measured before operation in 873 consecutive patients who underwent mitral valve surgery for mitral regurgitation between January 2002 and January 2010. PH was classified as none (sPAP $<40 \mathrm{~mm} \mathrm{Hg}$ ), mild $(40 \leq \mathrm{sPAP}<50 \mathrm{~mm} \mathrm{Hg}$ ), moderate $(50 \leq \mathrm{sPAP}<60 \mathrm{~mm} \mathrm{Hg}$ ), or severe ( $\mathrm{sPAP} \geq 60 \mathrm{~mm} \mathrm{Hg}$ ).
\end{abstract}

\begin{abstract}
Results: Increased preoperative sPAP was associated with greater left ventricular dysfunction and dilation, left atrial enlargement, more atrial fibrillation, and tricuspid regurgitation. Operative mortality was correlated with the degree of preoperative $\mathrm{PH}(2 \%, 3 \%, 8 \%$, and $12 \%$ for none, mild, moderate, and severe $\mathrm{PH}$, respectively, $P<.0001$ ). Long-term survival was related to preoperative sPAP (5-year survival: $88 \%, 79 \%, 65 \%$, and $53 \%$ for none, mild, moderate, and severe $\mathrm{PH}$, respectively; $P<.0001$ ). In multivariable analyses, sPAP was a predictor of both operative mortality (odds ratio, 1.023 per $1 \mathrm{~mm} \mathrm{Hg}$ increase; 95\% confidence interval, 1.003-1.044; $P=.0270$ ) and late death (hazard ratio, 1.018 per $1 \mathrm{~mm} \mathrm{Hg}$ increase; $95 \%$ confidence interval, $1.007-1.028$; $P=.001)$. Among 284 patients with isolated degenerative mitral regurgitation due to leaflet prolapse, actuarial survival was $97.5 \%, 91.2 \%$, and $80.5 \%$ for none, mild, and moderate to severe $\mathrm{PH}$, respectively $(P=.0002)$.

Conclusions: Preoperative sPAP is a powerful predictor of early and late survival after mitral valve operation for mitral regurgitation. Even modest increases in sPAP adversely affect outcomes. Mitral valve operation should be performed before the development of PH. (J Thorac Cardiovasc Surg 2011;142:1439-52)
\end{abstract}

Pulmonary hypertension (PH) is common among patients referred for mitral valve (MV) surgery for mitral regurgitation (MR). Although it is generally acknowledged that the presence of $\mathrm{PH}$ has a negative impact on operative outcomes, available data are remarkably scant and generally from small series. ${ }^{1-9}$ Current American College of Cardiology/American Heart Association and European Society for Cardiology guidelines recommend surgery for asymptomatic patients with MR and resting systolic pulmonary artery pressure (sPAP) greater than $50 \mathrm{~mm} \mathrm{Hg}$ as a class IIa recommendation based on level $\mathrm{C}$ evidence (expert opinion). ${ }^{10,11}$ Pulmonary artery pressure is not included in Society of Thoracic Surgeons risk models for

\footnotetext{
From the Division of Cardiac Surgery ${ }^{\mathrm{a}}$ and Department of Cardiology, ${ }^{\mathrm{b}}$ University of Maryland School of Medicine, Baltimore, Md; and Department of Statistics, ${ }^{\mathrm{c}}$ West Virginia University, Morgantown, WVa.

Disclosures: Authors have nothing to disclose with regard to commercial support.

Read at the 91st Annual Meeting of the American Association for Thoracic Surgery, Philadelphia, Pennsylvania, May 7-11, 2011.

Received for publication May 8, 2011; revisions received Aug 3, 2011; accepted for publication Aug 25, 2011; available ahead of print Oct 3, 2011.

Address for reprints: James S. Gammie, MD, Division of Cardiac Surgery, University of Maryland School of Medicine, N4W94, 22 S Greene St, Baltimore, MD 21201

(E-mail: jgammie@smail.umaryland.edu).

$0022-5223 / \$ 36.00$

Copyright (c) 2011 by The American Association for Thoracic Surgery

doi:10.1016/j.jtcvs.2011.08.030
}

valve surgery. ${ }^{12}$ The aim of the current study is to examine the impact of $\mathrm{PH}$ on early and long-term survival in a large cohort of patients undergoing surgery for MR.

\section{PATIENTS AND METHODS}

From January 2002 to January 2010, 953 patients underwent MV surgery for MR at the University of Maryland Medical Center. Patients were identified using the local Society of Thoracic Surgeons clinical database. This retrospective study was approved by the University of Maryland institutional review board with patient consent waived (HP-00046760). Preoperative, operative, and postoperative variables were collected. Patients with mitral stenosis, primary $\mathrm{PH}$, and unrecorded SPAP (s) were excluded, leaving 873 patients for analysis (Figure 1). To assess the impact of $\mathrm{PH}$ on patients undergoing primary operation for isolated degenerative MR with leaflet prolapse, ${ }^{13}$ we examined a subset of 284 patients undergoing primary isolated MV operation for leaflet prolapse (Figure 1). In cases in which preoperative right heart catheterization (RHC) was performed, the right atrial pressures, right ventricular pressures, pulmonary artery pressures (PAPs), and pulmonary capillary wedge pressures were recorded. If RHC was not performed, preoperative sPAP was measured with Doppler echocardiography (modified Bernoulli equation: $4 \times$ [tricuspid regurgitation jet velocity $]^{2}+$ right atrial pressure $[10 \mathrm{~mm} \mathrm{Hg}]$ ). sPAP was considered equal to right ventricular systolic pressure in the absence of pulmonary stenosis or right ventricular outflow tract obstruction. ${ }^{14}$ In cases in which the sPAP was available from RHC and Doppler echocardiography, the RHC results were used. The left ventricular function and diameters and left atrial (LA) dimensions and morphology of underlying MV disease were determined using Doppler echocardiography. The severity of valvular regurgitation was assessed semiquantitatively on a scale of 0 to $4(0=$ none or trace, 


$$
\begin{aligned}
& \text { Abbreviations and Acronyms } \\
& \begin{aligned}
\text { CI } & \text { confidence interval } \\
\text { LA } & =\text { left atrial } \\
\text { MR } & =\text { mitral regurgitation } \\
\text { MV } & \text { mitral valve } \\
\text { NYHA } & =\text { New York Heart Association } \\
\text { OR } & =\text { odds ratio } \\
\text { PH } & \text { pulmonary hypertension } \\
\text { RHC } & =\text { right heart catheterization } \\
\text { SBP } & \text { systolic blood pressure } \\
\text { SPAP } & =\text { systolic pulmonary artery pressure }
\end{aligned}
\end{aligned}
$$

$1=$ mild, $2=$ moderate, $3=$ moderate to severe, $4=$ severe).${ }^{15} \mathrm{PH}$ was defined as sPAP $40 \mathrm{~mm} \mathrm{Hg}$ or greater. Patients were categorized as follows: no $\mathrm{PH}$ ( $\mathrm{sPAP}<40 \mathrm{~mm} \mathrm{Hg}$ ), mild PH $(40 \mathrm{~mm} \mathrm{Hg} \leq \mathrm{sPAP}<50 \mathrm{~mm} \mathrm{Hg}$ ), moderate $\mathrm{PH}(50 \mathrm{~mm} \mathrm{Hg} \leq \mathrm{sPAP}<60 \mathrm{~mm} \mathrm{Hg})$, and severe $\mathrm{PH}$ (sPAP $\geq 60 \mathrm{~mm} \mathrm{Hg}$ ). The ratio of SPAP over systemic systolic blood pressure (sBP) was also measured. The mechanism of MR was determined on the basis of preoperative echocardiography and intraoperative inspection. ${ }^{16}$ Operative mortality was defined as in-hospital mortality or death within 30 days of operation. Predischarge echocardiography was routinely performed in a core echocardiography laboratory. Follow-up data including vital status and postoperative echocardiograms were obtained. Follow-up echocardiography was obtained at as many time points as available for each patient. A total of 1161 postoperative echocardiographic reports were obtained from our echocardiographic laboratory or the patient's referring cardiologist. SPAP data were analyzed during the following time periods: preoperatively, at discharge, during the first 2 years after surgery, and after 2 years. The Social Security Death Master File was used to determine vital status. ${ }^{17}$ The status of all patients was assessed cross-sectionally in May 2010. The mean follow-up time was $35 \pm 24$ months, and the mean duration of echocardiographic follow-up was $26 \pm 21$ months.

The following questions were addressed:

1. What are the predictors of preoperative $\mathrm{PH}$ ?

2. Did the presence of $\mathrm{PH}$ affect operative mortality and morbidity after MV operation?

3. What is the long-term survival after MV operation for MR in patients with and without preoperative $\mathrm{PH}$ ?

4. Does PH predict adverse short- and long-term outcome after MV operation for isolated degenerative MR?

5. Did pulmonary pressures regress over time after operation?

6. What is the best systolic pulmonary pressure cutoff point to minimize early and late mortality after MV operation for MR?

\section{Statistical Analysis}

Continuous variables are expressed as the mean \pm standard deviation (for reasonably symmetric variables) or median with the interquartile range (for clearly asymmetric variables). The values of categoric variables are expressed as proportions. Baseline compressions between patients' characteristics in different $\mathrm{PH}$ grades were performed using the chi-square test, Fisher exact test, Student $t$ test, Wilcoxon rank-sum test, and 1-way analysis of variance where appropriate. In cohort 2 (patients with isolated degenerative $\mathrm{MR}$ ), patients with moderate or severe $\mathrm{PH}$ were combined because of the small number of both groups. Logistic regression analysis was used (with variables listed in Appendix 1) to identify predictors of $\mathrm{PH}$ and operative mortality with a liberal retention criteria $(P<.10$ to enter and $P>.10$ to remove). The predicted probabilities of preoperative $\mathrm{PH}$ and operative mortality were identified. Long-term survival was evaluated with the
Kaplan-Meier method, and the log-rank test was used to compare the groups. A Cox proportional hazard regression analysis was performed for the determination of factors independently associated with long-term survival. The odds ratio $(\mathrm{OR})$ and hazard ratio $(\mathrm{HR})$ for $\mathrm{SPAP}$ as a continuous variable are displayed for $1 \mathrm{~mm} \mathrm{Hg}$ increment. Receiver operating characteristic curve analysis was performed to determine the cutoff values that best predict early and late survival. Analysis was performed using JMP 8.0 statistical software (SAS Institute Inc, Cary, NC). All statistic tests were 2 -sided, with an $\alpha$ level less than 0.05 for statistical significance.

\section{RESULTS \\ Patient Population}

Between January 2002 and January 2010, 953 consecutive MV operations were performed at a single center. From this total, 888 patients met the inclusion criteria (Figure 1). sPAP was measured in 873 patients (98\%) using RHC $(591,68 \%)$ and echocardiography $(282,32 \%)$. The mean patient age was $59 \pm 14$ years. A total of 493 patients $(57 \%)$ were in New York Heart Association (NYHA) functional class III or IV, and 260 patients (30\%) had atrial fibrillation (Table 1). Moderate-to-severe or severe degree of MR was present in 775 patients $(89 \%)$. Right ventricular dysfunction defined as any degree of impairment in right ventricular contraction was present in 137 of 873 patients $(15 \%)$.

\section{Preoperative Characteristics and Pulmonary Artery Pressure}

sPAP was determined using RHC in 591 patients $(68 \%)$ (performed a mean of $29 \pm 45$ days before operation). Average sPAP, mean pulmonary artery pressure, right atrial pressure, right ventricular pressure, and pulmonary capillary wedge pressure measured during RHC were $43 \pm 17$ $\mathrm{mm} \mathrm{Hg}, 29 \pm 12 \mathrm{~mm} \mathrm{Hg}, 9 \pm 6 \mathrm{~mm} \mathrm{Hg}, 11 \pm 8 \mathrm{~mm} \mathrm{Hg}$, and $18 \pm 9 \mathrm{~mm} \mathrm{Hg}$, respectively. sPAP was recorded on preoperative echocardiography in 621 patients $(71 \%)$ (performed a mean of $35 \pm 48$ days before operation) with a mean sPAP of $46 \pm 15 \mathrm{~mm} \mathrm{Hg}$. sPAP was measured in 373 patients with both echocardiography (mean \pm standard deviation, $47 \pm 16 \mathrm{~mm} \mathrm{Hg}$ ) and RHC (mean \pm standard deviation, $45 \pm 17 \mathrm{~mm} \mathrm{Hg}$ ). Measured mean sPAPs were not significantly different between these 2 modalities $(P=.9)$. A linear relationship was observed between the sPAPs recorded with echocardiography and RHC $(r=0.39$, $P<.0001)$.

PH (sPAP $\geq 40 \mathrm{~mm} \mathrm{Hg}$ ) was present in 466 patients $(53 \%)$ and was severe (sPAP $\geq 60 \mathrm{~mm} \mathrm{Hg}$ ) in 148 patients $(17 \%)$ (Table 2). We observed a temporal trend toward a greater percentage of patients with no (sPAP $<40 \mathrm{~mm}$ $\mathrm{Hg}$ ) or mild ( $40 \leq \mathrm{sPAP}<50 \mathrm{~mm} \mathrm{Hg}$ ) PH undergoing surgery over the course of the study. Higher preoperative sPAPs were associated with increased age, female sex, higher NYHA function class, and a greater prevalence of comorbidities (Table 2). The mean left ventricular ejection fraction decreased as SPAP increased, whereas the mean left ventricular end-diastolic dimension was similar across 


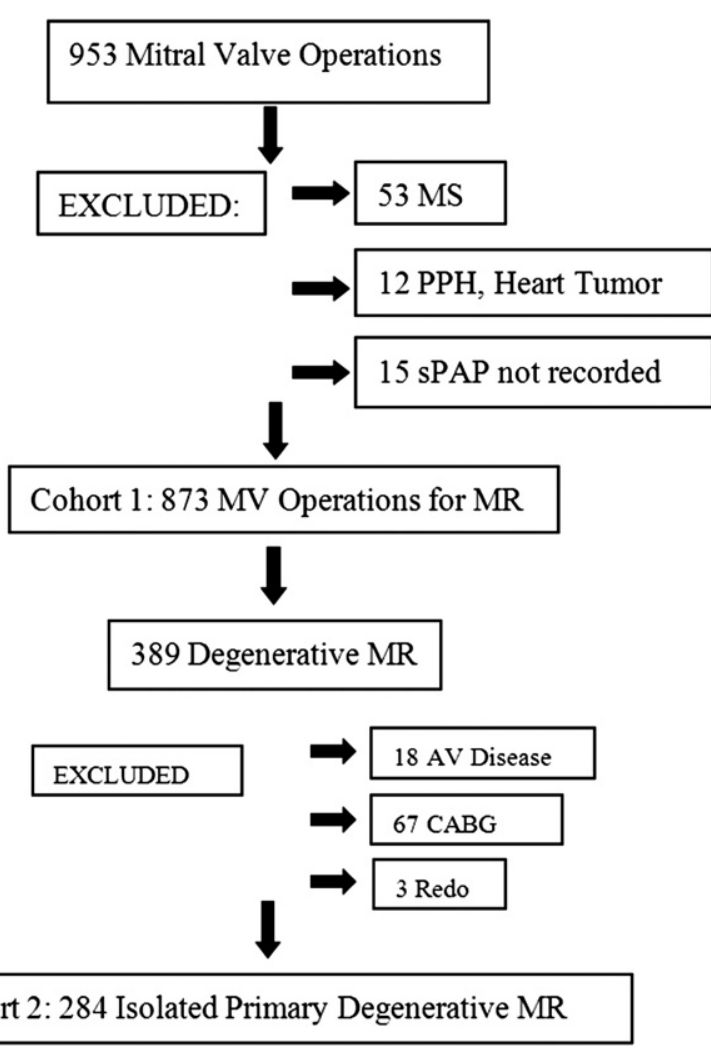

FIGURE 1. Patient population: exclusion criteria and final analysis population for cohort 1 and 2 studies. $A V$, Aortic valve; $C A B G$, coronary artery bypass graft surgery; $M R$, mitral regurgitation; $M S$, mitral stenosis; $M V$, mitral valve; $P P H$, primary pulmonary hypertension; Redo, redo cardiac surgery; $s P A P$, systolic pulmonary artery pressure.

varying levels of sPAP. Greater degrees of LA enlargement, more atrial fibrillation, tricuspid regurgitation, systemic hypertension, and RV dysfunction were present in patients with higher preoperative SPAP. By multivariable analysis, predictors that were significantly associated with higher preoperative sPAP included female gender (OR, 2.88; 95\% confidence interval $[\mathrm{CI}], 1.92-4.54 ; P<.0001)$, LA dimension (OR, 1.05 per $1-\mathrm{mm}$ increase; $95 \%$ CI, $1.03-$ 1.08; $P<.0001)$, left ventricular end-systolic dimension (OR, 1.05 per $1-\mathrm{mm}$ increase; 95\% CI, 1.03-1.08; $P<.0001)$, chronic lung disease (OR, 3.40; 95\% CI, $1.70-7.70 ; P=.0005)$, dialysis (OR, 7.86; 95\% CI, 3.3233.38; $P<.0001$ ), previous myocardial infarction (OR, $2.37 ; 95 \%$ CI, $1.52-4.80 ; P=.0028)$, and body mass index (OR, 1.05 per $1 \mathrm{~kg} / \mathrm{m}^{2}$ increase; $95 \% \mathrm{CI}, 1.02-1.09$; $P=$.0017) (C-statistic $=0.768)$.

\section{Operative Characteristics}

Concomitant procedures performed included coronary artery bypass in 253 patients (29\%), tricuspid valve operation in 133 patients $(15 \%)$, aortic valve operation in 100 patients $(11 \%)$, and CryoMaze procedure in 185 patients
TABLE 1. Selected preoperative patient characteristics for 873 patients undergoing mitral valve operation for mitral regurgitation

\begin{tabular}{|c|c|}
\hline Characteristic & No. $(\%)$, or mean \pm SD \\
\hline Age (y) & $59 \pm 14$ \\
\hline Female gender & $361(41 \%)$ \\
\hline BMI $\left(\mathrm{kg} / \mathrm{m}^{2}\right)$ & $27 \pm 7$ \\
\hline NYHA class III/IV & $493(57 \%)$ \\
\hline Atrial fibrillation & $260(30 \%)$ \\
\hline Prior cardiac surgery & $61(7 \%)$ \\
\hline Prior MV surgery & $22(3 \%)$ \\
\hline Chronic lung disease & $115(13 \%)$ \\
\hline Hypertension & $561(64 \%)$ \\
\hline Diabetes mellitus & $157(18 \%)$ \\
\hline Renal failure (dialysis) & $50(6 \%)$ \\
\hline \multicolumn{2}{|l|}{ Cardiac structure, function } \\
\hline LV ejection fraction, mean, $\%$ & $52 \pm 14$ \\
\hline LA diameter, $\mathrm{cm}$ & $4.6 \pm 0.8\left(n^{*}=641\right)$ \\
\hline LV end-diastolic diameter, $\mathrm{cm}$ & $5.4 \pm 0.8(\mathrm{n}=656)$ \\
\hline LV end-systolic diameter, $\mathrm{cm}$ & $3.8 \pm 0.9(\mathrm{n}=544)$ \\
\hline \multicolumn{2}{|l|}{ sPAP (mm Hg) } \\
\hline Echocardiography & $46 \pm 15(n=621)$ \\
\hline RHC & $43 \pm 17(n=591)$ \\
\hline RV dysfunction & $137(15 \%)$ \\
\hline \multicolumn{2}{|l|}{ MR grade } \\
\hline Moderate & $98(11 \%)$ \\
\hline Moderate-to-severe & $210(24 \%)$ \\
\hline Severe & $565(65 \%)$ \\
\hline \multicolumn{2}{|l|}{ TR grade } \\
\hline None/mild & $664(76 \%)$ \\
\hline Moderate & $114(13 \%)$ \\
\hline Moderate-to-severe & $36(4 \%)$ \\
\hline Severe & $59(7 \%)$ \\
\hline \multicolumn{2}{|l|}{ AI grade } \\
\hline None/mild & $787(90 \%)$ \\
\hline Moderate & $44(5 \%)$ \\
\hline Severe & $42(5 \%)$ \\
\hline Severe AS & $49(6 \%)$ \\
\hline
\end{tabular}

$A I$, Aortic insufficiency; $A S$, aortic stenosis; $A V$, aortic valve; $B M I$, body mass index $L A$, left atrium; $L V$, left ventricle; $M R$, mitral regurgitation; $M V$, mitral valve; $N Y H A$, New York Heart Association; $R H C$, right heart catheterization; $R V$, right ventricle; $S D$, standard deviation; $T R$, tricuspid regurgitation; $S P A P$, systolic pulmonary artery pressure. *Data available.

(21\%). Sixty-one patients $(7 \%)$ underwent redo operation (Table 3). MV repair was performed in 747 patients $(86 \%)$, and MV replacement was performed in 126 patients (14\%). Operative characteristics as a function of preoperative sPAP are summarized in Table 4. Patients with higher sPAP more commonly required concomitant procedures. MV repair was less commonly performed as the degree of $\mathrm{PH}$ increased (repair rate $92 \%$ for no $\mathrm{PH}, 88 \%$ for mild $\mathrm{PH}, 82 \%$ for moderate $\mathrm{PH}$, and $70 \%$ for severe $\mathrm{PH}$, $P<.0001$ ) (Table 4).

\section{Operative Outcomes}

The operative mortality rate for the entire population was $5 \%(\mathrm{n}=43$, in hospital $=40)$. Operative mortality was $2 \%$ 
TABLE 2. Baseline characteristics of the patient population according to degree of pulmonary hypertension*

\begin{tabular}{|c|c|c|c|c|c|}
\hline Variable & $\begin{array}{c}\text { sPAP }<40 \mathbf{~ m m ~ H g} \\
\mathbf{4 0 7}(\mathbf{4 7} \%) \\
\end{array}$ & $\begin{array}{c}40 \leq \mathbf{s P A P}<50 \mathrm{~mm} \mathrm{Hg} \\
176(20 \%)\end{array}$ & $\begin{array}{c}50 \leq \text { sPAP }<60 \mathrm{~mm} \mathrm{Hg} \\
142(16 \%)\end{array}$ & $\begin{array}{c}\text { sPAP } \geq 60 \mathrm{~mm} \mathrm{Hg} \\
148(17 \%) \\
\end{array}$ & $P$ value \\
\hline \multicolumn{6}{|l|}{ sPAP (mm Hg) } \\
\hline Mean \pm SD & $30 \pm 6$ & $44 \pm 3$ & $53 \pm 3$ & $71 \pm 10$ & $<.00001$ \\
\hline Median (25th-75th percentile) & $30(25-35)$ & $44(41-46)$ & $53(50-55)$ & $69(63-77)$ & \\
\hline sPAP/sBP $($ mean $\pm \mathrm{SD})$ & $0.24 \pm 0.05$ & $0.34 \pm 0.06$ & $0.43 \pm 0.10$ & $0.56 \pm 0.12$ & $<.0001$ \\
\hline Age (y) & $58 \pm 14$ & $61 \pm 13$ & $61 \pm 15$ & $62 \pm 14$ & .0048 \\
\hline Female gender & $144(35 \%)$ & $82(47 \%)$ & $78(45 \%)$ & $71(48 \%)$ & .0008 \\
\hline $\mathrm{BMI}\left(\mathrm{kg} / \mathrm{m}^{2}\right.$, mean $\left.\pm \mathrm{SD}\right)$ & $27 \pm 6$ & $28 \pm 8$ & $28 \pm 6$ & $29 \pm 8$ & .0005 \\
\hline Morbid obesity $\dagger$ & $16(16 \%)$ & $18(31 \%)$ & $8(14 \%)$ & $16(28 \%)$ & .2274 \\
\hline NYHA class III/IV & $188(46 \%)$ & $102(58 \%)$ & $96(68 \%)$ & $107(72 \%)$ & .0001 \\
\hline Atrial fibrillation & $100(25 \%)$ & $63(36 \%)$ & $51(36 \%)$ & $56(38 \%)$ & .00034 \\
\hline $\operatorname{LVEF}(\%)$ & $55 \pm 13$ & $52 \pm 14$ & $47 \pm 16$ & $50 \pm 14$ & .0001 \\
\hline LVEDD (cm) & $5.4 \pm 0.7$ & $5.5 \pm 0.9$ & $5.5 \pm 0.9$ & $5.5 \pm 0.9$ & .606 \\
\hline $\operatorname{LVESD}(\mathrm{cm})$ & $3.6 \pm 0.8$ & $3.9 \pm 0.1$ & $4.1 \pm 0.1$ & $4.1 \pm 0.1$ & .0001 \\
\hline LA diameter $(\mathrm{cm})$ & $4.4 \pm 0.8$ & $4.6 \pm 0.9$ & $4.8 \pm 0.7$ & $5.0 \pm 0.8$ & .0001 \\
\hline RV dysfunction & $19(5 \%)$ & $22(13 \%)$ & $43(30 \%)$ & $53(36 \%)$ & .0001 \\
\hline MR grade & $3.5 \pm 0.7$ & $3.2 \pm 0.8$ & $3.4 \pm 0.8$ & $3.6 \pm 0.6$ & .0003 \\
\hline TR grade & $0.6 \pm 0.8$ & $1.05 \pm 0.9$ & $1.4 \pm 1.1$ & $1.8 \pm 1.3$ & $<.0001$ \\
\hline AI grade & $0.2 \pm 0.7$ & $0.4 \pm 1$ & $0.5 \pm 1$ & $0.6 \pm 1$ & .0002 \\
\hline Severe AS & $10(2 \%)$ & $8(5 \%)$ & $16(11 \%)$ & $15(10 \%)$ & $<.0001$ \\
\hline Chronic lung disease & $36(9 \%)$ & $28(16 \%)$ & $21(15 \%)$ & $30(21 \%)$ & .0021 \\
\hline Redo operation & $21(5 \%)$ & $11(6 \%)$ & $12(8 \%)$ & $17(11 \%)$ & .0625 \\
\hline Diabetes mellitus & $45(11 \%)$ & $37(21 \%)$ & $27(19 \%)$ & $48(32 \%)$ & .0001 \\
\hline Hypertension & $225(55 \%)$ & $122(69 \%)$ & $100(70 \%)$ & $114(77 \%)$ & .0001 \\
\hline Peripheral arterial disease & $20(5 \%)$ & $8(5 \%)$ & $13(9 \%)$ & $17(11 \%)$ & .041 \\
\hline History of stroke & $30(7 \%)$ & $19(11 \%)$ & $21(15 \%)$ & $16(11 \%)$ & .071 \\
\hline Previous MI & $41(10 \%)$ & $36(20 \%)$ & $30(21 \%)$ & $31(21 \%)$ & .0003 \\
\hline Cerebrovascular disease & $44(11 \%)$ & $24(14 \%)$ & $25(18 \%)$ & $29(20 \%)$ & .031 \\
\hline Renal failure (dialysis) & $6(1 \%)$ & $6(3 \%)$ & $18(13 \%)$ & $20(14 \%)$ & .0001 \\
\hline ACE inhibitors/ARB & $161(40 \%)$ & $70(40 \%)$ & $76(54 \%)$ & $64(43 \%)$ & .0284 \\
\hline Beta-blockers & $176(43 \%)$ & $96(56 \%)$ & $85(60 \%)$ & $91(61 \%)$ & .0009 \\
\hline
\end{tabular}

Data presented in mean $\pm \mathrm{SD}$ or n (\%). $A I$, Aortic insufficiency; $A C E$, angiotensin-converting enzyme; $A R B$, angiotensin receptor blocker; $A S$, aortic stenosis; $B M I$, body mass index; $L A$, left atrium; $L V E F$, left ventricular ejection fraction; $L V E D D$, left ventricular end-diastolic diameter; $L V E S D$, left ventricular end-diastolic diameter; $M I$, myocardial infarction; $M R$, mitral regurgitation; NYHA, New York Heart Association; $R V$, right ventricle; $s B P$, systemic blood pressure in systole; $S D$, standard deviation; $s P A P$, systolic pulmonary artery pressure; $T R$, tricuspid regurgitation. ${ }^{*} \mathrm{PH}$ absent: sPAP $<40 \mathrm{~mm} \mathrm{Hg}$; mild $\mathrm{PH}: 40 \leq \mathrm{sPAP}<50 \mathrm{~mm} \mathrm{Hg}$; moderate PH: $50 \leq \mathrm{sPAP}<60 \mathrm{~mm} \mathrm{Hg}$; severe PH: sPAP $\geq 60 \mathrm{~mm} \mathrm{Hg}$. †Morbid obesity: BMI $\geq 40 \mathrm{~kg} / \mathrm{m}^{2}$.

for patients with no $\mathrm{PH}(\mathrm{sPAP}<40 \mathrm{~mm} \mathrm{Hg}$ ) compared with $3 \%$ for patients with mild $\mathrm{PH}(40 \leq \mathrm{sPAP}<50 \mathrm{~mm} \mathrm{Hg})$, $8 \%$ for moderate $\mathrm{PH}(50 \leq \mathrm{sPAP}<60 \mathrm{~mm} \mathrm{Hg})$, and $12 \%$ for those with severe preoperative PH (sPAP $\geq 60$ $\mathrm{mm} \mathrm{Hg})(P<.0001)$ (Figure 2). By multivariable analysis, preoperative sPAP was a predictor associated with operative mortality (Table 5).

Major operative morbidity for the entire group included new-onset atrial fibrillation $(121,14 \%)$, dialysis (31, $3.5 \%)$, stroke $(19,2 \%)$, and sepsis $(22,2.5 \%)$. Higher preoperative SPAPs were associated with higher rates of postoperative sepsis, prolonged ventilation, and dialysis (Table 4). On comparison of patients with no $\mathrm{PH}$ $($ sPAP $<40 \mathrm{~mm} \mathrm{Hg}$ ) with those with mild $\mathrm{PH}(40 \leq$ sPAP $<50 \mathrm{~mm} \mathrm{Hg}$ ), morbidity, including renal failure (dialysis) $(P=.8)$, atrial fibrillation $(P=.24)$, and stroke $(P=.8)$, was not significantly different. However, prolonged ventilation (no $\mathrm{PH}: 43$ [11\%] vs mild $\mathrm{PH}: 44$
[25\%], $P<.0001$ ) and longer hospital stay (median 5 days for no $\mathrm{PH}$ vs 8 days for mild $\mathrm{PH}, P<.0001$ ) and intensive care unit stay more than 24 hours (no PH: 13 [3\%] vs mild PH $10[6 \%], P=.0244$ ) were more common in patients with mild PH (Table 4).

\section{Late Survival}

Actuarial survival in patients without $\mathrm{PH}$ ( $\mathrm{SPAP}<40 \mathrm{~mm}$ $\mathrm{Hg}$ ) at $1,3,5$, and 7 years was $96 \%, 92 \%, 86 \%$, and $84 \%$, respectively, compared with $83 \%, 73 \%, 67 \%$, and $61 \%$, respectively, for those with preoperative $\mathrm{PH}$ (sPAP $\geq 40$ $\mathrm{mm} \mathrm{Hg})(P<.00279)$ (Figure 3, A). Long-term survival progressively decreased with increasing preoperative sPAP grade $(P<.0001)$ (Figure $3, B)$. In a Cox proportional hazards analysis, increasing preoperative sPAP was associated with decreased long-term survival (Table 6). Among patients with no or minimal preoperative symptoms (380 [43\%]) (NYHA class I: 88 [10\%], NYHA class II: 292 
TABLE 3. Operative characteristics for 873 patients undergoing mitral valve operation for mitral regurgitation

\begin{tabular}{|c|c|}
\hline Characteristic & No. $(\%)$, or mean \pm SD \\
\hline \multicolumn{2}{|l|}{ MV operation } \\
\hline Repair & $747(86 \%)$ \\
\hline Replacement & $126(14 \%)$ \\
\hline \multicolumn{2}{|l|}{ MV pathology } \\
\hline $\begin{array}{l}\text { Degenerative MR due to leaflet } \\
\text { prolapse }\end{array}$ & $389(44 \%)$ \\
\hline Anterior leaflet prolapse & $52(13 \%)$ \\
\hline Posterior leaflet prolapse & $270(70 \%)$ \\
\hline Bileaflet prolapse & $67(17 \%)$ \\
\hline $\begin{array}{l}\text { Isolated annular dilation (type I) or } \\
\text { functional (type IIIb } \pm \text { type I) }\end{array}$ & $271(31 \%)$ \\
\hline Rheumatic & $70(8 \%)$ \\
\hline Endocarditis & $132(15 \%)$ \\
\hline Paravalvular leak & $8(1 \%)$ \\
\hline Prosthetic valve degeneration & $3(1 \%)$ \\
\hline Redo operation & $61(7 \%)$ \\
\hline \multicolumn{2}{|l|}{ Concomitant procedures } \\
\hline Coronary artery bypass & $253(29 \%)$ \\
\hline TV operation & $133(15 \%)$ \\
\hline AV operation & $100(11 \%)$ \\
\hline CryoMaze procedure & $185(21 \%)$ \\
\hline ASD closure & $22(3 \%)$ \\
\hline \multicolumn{2}{|l|}{ Perfusion time (min) } \\
\hline Mean & $126 \pm 49$ \\
\hline Median & 117 \\
\hline \multicolumn{2}{|l|}{ Crossclamp time (min) } \\
\hline Mean & $97 \pm 37$ \\
\hline Median & 94 \\
\hline Operative mortality & $43(5 \%)$ \\
\hline
\end{tabular}

[33\%]), even a mild increase in preoperative sPAP $(40 \leq$ sPAP $<50 \mathrm{~mm} \mathrm{Hg}$ ) compromised long-term survival (log rank, $P=.0004)$. Among those 373 patients with sPAP determined with both echocardiography and RHC, we found that echocardiographic-derived SPAP was similarly predictive of late mortality as was sPAP determined by RHC (echocardiographic-derived sPAP: HR, 1.024 per $1 \mathrm{~mm}$ Hg increment; 95\% CI, 1.011-1.034; $P=.0004$ vs RHCderived sPAP: HR, 1.029 per $1 \mathrm{~mm} \mathrm{Hg}$ increment; 95\% CI, $1.0117-1.041 ; P<.0001)$.

Even after excluding patients with a history of myocardial infarction $(\mathrm{n}=138)$, concomitant aortic valve surgery $(\mathrm{n}=100)$, or coronary artery bypass surgery $(\mathrm{n}=253)$, patients with SPAP $40 \mathrm{~mm} \mathrm{Hg}$ or greater had higher operative mortality and long-term death (Appendix 2).

\section{Analysis of Outcomes Based on the Ratio of Systolic Pulmonary Artery Pressure to Systemic Blood Pressure}

On the basis of the ratio of systolic pulmonary to systolic systemic blood pressure, patients were categorized as having no $\mathrm{PH}(\mathrm{sPAP} / \mathrm{sBP}<0.3)(\mathrm{n}=387,45 \%)$, mild $\mathrm{PH}(0.3 \leq \mathrm{sPAP} / \mathrm{sBP}<0.4)(\mathrm{n}=200,23 \%)$, moderate $\mathrm{PH}(0.4 \leq \mathrm{sPAP}<0.5)(\mathrm{n}=143,16 \%)$, and severe $\mathrm{PH}$ $(\mathrm{sPAP} / \mathrm{sBP} \geq 0.5)(\mathrm{n}=143,16 \%)$. Operative mortality was $2 \%(\mathrm{n}=8)$ for no $\mathrm{PH}, 4 \%(\mathrm{n}=8)$ for mild $\mathrm{PH}$, $6.3 \%(\mathrm{n}=9)$ for moderate $\mathrm{PH}$, and $13 \%(\mathrm{n}=18)$ for severe PH $(P<.0001)$. Long-term survival decreased significantly with increased preoperative sPAP/sBP ratio (Appendix Figure 1). These findings were nearly identical to those in which only isolated SPAP was used as a predictor.

\section{Isolated Degenerative Mitral Regurgitation}

Overall, 284 patients with isolated degenerative MR with leaflet prolapse were studied in cohort 2 . The mean age was $57 \pm 13$ years, and the mean sPAP was $36 \pm 14 \mathrm{~mm} \mathrm{Hg}$ (Table 7). Preoperative sPAP was measured using RHC in 214 patients $(75 \%)$. Preoperatively, 193 patients $(68 \%)$ had no PH (sPAP $<40 \mathrm{~mm} \mathrm{Hg}$ ), 48 patients $(17 \%)$ had mild $\mathrm{PH}(40 \leq$ sPAP $<50 \mathrm{~mm} \mathrm{Hg})$, and 43 patients $(15 \%)$ had moderate/severe PH (sPAP $\geq 50 \mathrm{~mm} \mathrm{Hg}$ ). Preoperative NYHA class III or IV symptoms were present in 121 patients (39\%), and 173 patients $(61 \%)$ had class I or II symptoms. MV repair was performed in 277 patients $(98 \%)$, and MV replacement was performed in 8 patients $(2 \%)$. Concomitant procedures, including tricuspid valve repair $(\mathrm{n}=18,6 \%)$ and CryoMaze procedure $(\mathrm{n}=58$, $20 \%$ ), were more common in patients with $\mathrm{PH}$ (sPAP $\geq$ $40 \mathrm{~mm} \mathrm{Hg}$ ). Operative mortality was $0.8 \%$ (2/284). Kaplan-Meier analysis showed that survival decreased with increasing preoperative PH grade (Figure 4). Overall actuarial survival at 1,3 , and 5 years after MV surgery was $99.5 \%, 98.5 \%$, and $97.5 \%$, respectively, in patients with no PH (sPAP $<40 \mathrm{~mm} \mathrm{Hg}$ ) compared with $95.8 \%$, $92.9 \%$, and $91.2 \%$, respectively, for those with mild $\mathrm{PH}$ $(40 \leq \mathrm{sPAP}<50 \mathrm{~mm} \mathrm{Hg})$ and $90.6 \%, 84.2 \%$, and $80.5 \%$, respectively, for those with moderate to severe $\mathrm{PH}$ $(\mathrm{sPAP} \geq 50 \mathrm{~mm} \mathrm{Hg})(P=.0002)$. Compared with patients with no $\mathrm{PH}, 5$-year survival decreased in patients with mild $\mathrm{PH}(98 \% \pm 1 \%$ for no $\mathrm{PH}$ vs $91 \% \pm 4 \%$ for mild $\mathrm{PH}$, $P=.002)$. In a Cox proportional hazards analysis, factors associated with decreased long-term survival included increasing preoperative SPAP (HR, 1.037 per $1 \mathrm{~mm} \mathrm{Hg}$ increase; 95\% CI, 1.004-1.066; $P=.0277$ ), age (HR, $1.303 ; 95 \%$ CI, $1.051-1.625 ; P=.0277)$, and left ventricular ejection fraction (HR, 0.902; 95\% CI, 0.854-0.956; $P=.001)($ C-statistic for the multivariable model $=0.9$ ).

\section{Pulmonary Artery Pressure Changes After Operation}

The mean sPAP at different time points is demonstrated in Figure 5, A. In the entire population, mean sPAP decreased modestly from $43 \pm 16 \mathrm{~mm} \mathrm{Hg}$ before operation to $39 \pm 11 \mathrm{~mm} \mathrm{Hg}$ before hospital discharge $(P<.0001)$. Compared with the predischarge value, mean sPAP was 
TABLE 4. Operative characteristics and hospital morbidity and mortality as a function of preoperative systolic pulmonary artery pressure*

\begin{tabular}{|c|c|c|c|c|c|}
\hline Variable & $\begin{array}{c}\text { sPAP }<40 \mathrm{~mm} \text { Hg } \\
407(47 \%)\end{array}$ & $\begin{array}{c}40 \leq \text { sPAP }<50 \mathrm{~mm} \mathrm{Hg} \\
\mathbf{1 7 6}(\mathbf{2 0} \%)\end{array}$ & $\begin{array}{c}50 \leq \text { sPAP }<60 \mathrm{~mm} \mathrm{Hg} \\
142(16 \%)\end{array}$ & $\begin{array}{c}\mathrm{sPAP} \geq 60 \mathrm{~mm} \mathrm{Hg} \\
148(17 \%)\end{array}$ & $P$ value \\
\hline $\mathrm{CVP} \dagger(\mathrm{mm} \mathrm{Hg})$ & $10 \pm 5$ & $12 \pm 5$ & $13 \pm 6$ & $15 \pm 6$ & $<.001$ \\
\hline \multicolumn{6}{|l|}{ MV pathology } \\
\hline Degenerative (leaflet prolapse) & $240(59 \%)$ & $70(40 \%)$ & $36(25 \%)$ & $43(29 \%)$ & $<.0001$ \\
\hline $\begin{array}{l}\text { Isolated annular dilation (type I) or } \\
\text { functional (type IIIb } \pm \text { type I) }\end{array}$ & $90(22 \%)$ & $62(35 \%)$ & $66(46 \%)$ & $53(36 \%)$ & $<.001$ \\
\hline Rheumatic & $21(55 \%)$ & $13(7 \%)$ & $13(9 \%)$ & $23(16 \%)$ & .001 \\
\hline Endocarditis & $51(13 \%)$ & $31(18 \%)$ & $25(18 \%)$ & $25(17 \%)$ & 282 \\
\hline MV repair & $373(92 \%)$ & $154(88 \%)$ & $117(82 \%)$ & $103(70 \%)$ & $<.0001$ \\
\hline \multicolumn{6}{|l|}{ Concomitant procedure } \\
\hline Coronary artery bypass & $89(22 \%)$ & $51(29 \%)$ & $56(39 \%)$ & $57(39 \%)$ & $<.0001$ \\
\hline TV operation & $21(5 \%)$ & $29(16 \%)$ & $30(21 \%)$ & $50(34 \%)$ & $<.0001$ \\
\hline AV operation & $27(7 \%)$ & $22(13 \%)$ & $23(16 \%)$ & $28(19 \%)$ & .0001 \\
\hline CryoMaze procedure & $71(17 \%)$ & $53(30 \%)$ & $30(21 \%)$ & $31(21 \%)$ & .0008 \\
\hline Operative mortality & $7(2 \%)$ & $6(3 \%)$ & $12(8 \%)$ & $18(12 \%)$ & $<.0001$ \\
\hline \multicolumn{6}{|l|}{ Morbidity } \\
\hline Renal failure (dialysis) & $8(2 \%)$ & $4(2 \%)$ & $9(6 \%)$ & $10(7 \%)$ & .0094 \\
\hline Prolonged ventilation & $43(11 \%)$ & $44(25 \%)$ & $35(25 \%)$ & $54(36 \%)$ & $<.0001$ \\
\hline Sepsis & $7(2 \%)$ & $1(1 \%)$ & $4(3 \%)$ & $10(7 \%)$ & .0022 \\
\hline Atrial fibrillation & $46(11 \%)$ & $26(15 \%)$ & $27(19 \%)$ & $22(15 \%)$ & .12 \\
\hline Stroke & $8(2 \%)$ & $3(2 \%)$ & $4(3 \%)$ & $4(3 \%)$ & .31 \\
\hline \multicolumn{6}{|l|}{ Length of stay } \\
\hline $\mathrm{ICU}>24 \mathrm{~h}$ & $13(3 \%)$ & $10(6 \%)$ & $8(6 \%)$ & $12(8 \%)$ & .021 \\
\hline LOS (median [25th-75th], d) & $5(4-9)$ & $8(6-16)$ & $11.5(7-19)$ & $12(8-22)$ & $<.0001$ \\
\hline
\end{tabular}

$A V$, Aortic valve; $C V P$, central venous pressure; $I C U$, intensive care unit; $L O S$, length of stay; $M V$, mitral valve; $s P A P$, systolic pulmonary artery pressure; $T V$, tricuspid valve. $*$ PH absent: sPAP < 40 mm Hg; mild PH: $40 \leq$ sPAP < 50 mm Hg; moderate PH: $50 \leq$ sPAP < 60 mm Hg; Severe PH: sPAP $\geq 60$ mm Hg. $\dagger$ Intraoperative, before incision.

not markedly changed within the first 2 years $(39 \pm 12 \mathrm{~mm}$ $\mathrm{Hg}, P=.907)$ and after 2 years of follow-up $(38 \pm 13 \mathrm{~mm}$ $\mathrm{Hg}, P=.952$ ) (Figure 5, $A$ ). Figure 5, $B$, demonstrates sPAP at different time points for each category of pulmonary artery pressure. Compared with preoperative values, SPAP decreased on predischarge echocardiography in all categories except in patients with no PH where there was a small increase. The relative decrease was most pronounced in patients with preoperative moderate $(50 \leq \mathrm{sPAP}<60 \mathrm{~mm}$ $\mathrm{Hg}$ ) and severe PH (sPAP $\geq 60 \mathrm{~mm} \mathrm{Hg}$ ). On late follow-

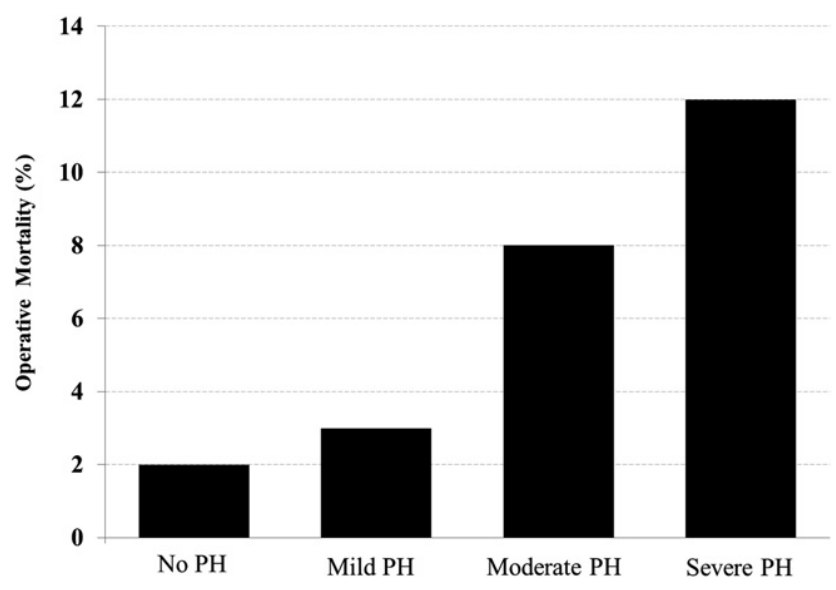

FIGURE 2. Hospital mortality according to preoperative $\mathrm{PH}$ grade $(P<.0001) . P H$, Pulmonary hypertension. up, sPAP was similar to predischarge levels. Residual PH (sPAP $\geq 40 \mathrm{~mm} \mathrm{Hg}$ ) was more commonly present among patients with mild ( $40 \leq$ sPAP $<50 \mathrm{~mm} \mathrm{Hg}$ ), moderate $(50 \leq \mathrm{sPAP}<60 \mathrm{~mm} \mathrm{Hg}$ ), or severe degree (sPAP $\geq 60$ $\mathrm{mm} \mathrm{Hg}$ ) of preoperative $\mathrm{PH}$ (Figure 5, $B$ and $C$ ). Of 159 patients who had preoperative sPAP $50 \mathrm{~mm} \mathrm{Hg}$ or greater and follow-up echocardiographic data available, $56(35 \%)$ still had sPAP $50 \mathrm{~mm} \mathrm{Hg}$ or greater 2 years (mean, $714 \pm 603$ days) after surgery.

\section{DISCUSSION \\ Principal Findings}

This article presents early and late outcomes after MV surgery in a large series of patients with MR according

TABLE 5. Predictors of operative mortality*

\begin{tabular}{lccc}
\hline \multicolumn{1}{c}{ Variable } & OR & $\mathbf{9 5} \%$ CI & $\boldsymbol{P}$ \\
\hline sPAP (per 1 mm Hg increment) & 1.023 & $1.003-1.044$ & .0270 \\
NYHA functional class III-IV & 3.442 & $1.040-1.410$ & .0052 \\
Age (per unit) & 1.034 & $1.007-1.064$ & .0159 \\
Concomitant CABG & 2.133 & $1.041-4.464$ & .0395 \\
Dialysis & 4.451 & $1.068-1.785$ & .0018 \\
IE & 3.017 & $1.204-7.407$ & .0194 \\
RV dysfunction & 3.178 & $1.552-6.488$ & .0017 \\
\hline
\end{tabular}

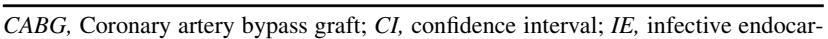
ditis; NYHA, New York Heart Association; $O R$, odds ratio; $R V$, right ventricle; $s P A P$, systolic pulmonary artery pressure. ${ }^{*} \mathrm{C}$-statistic $=0.854$. 

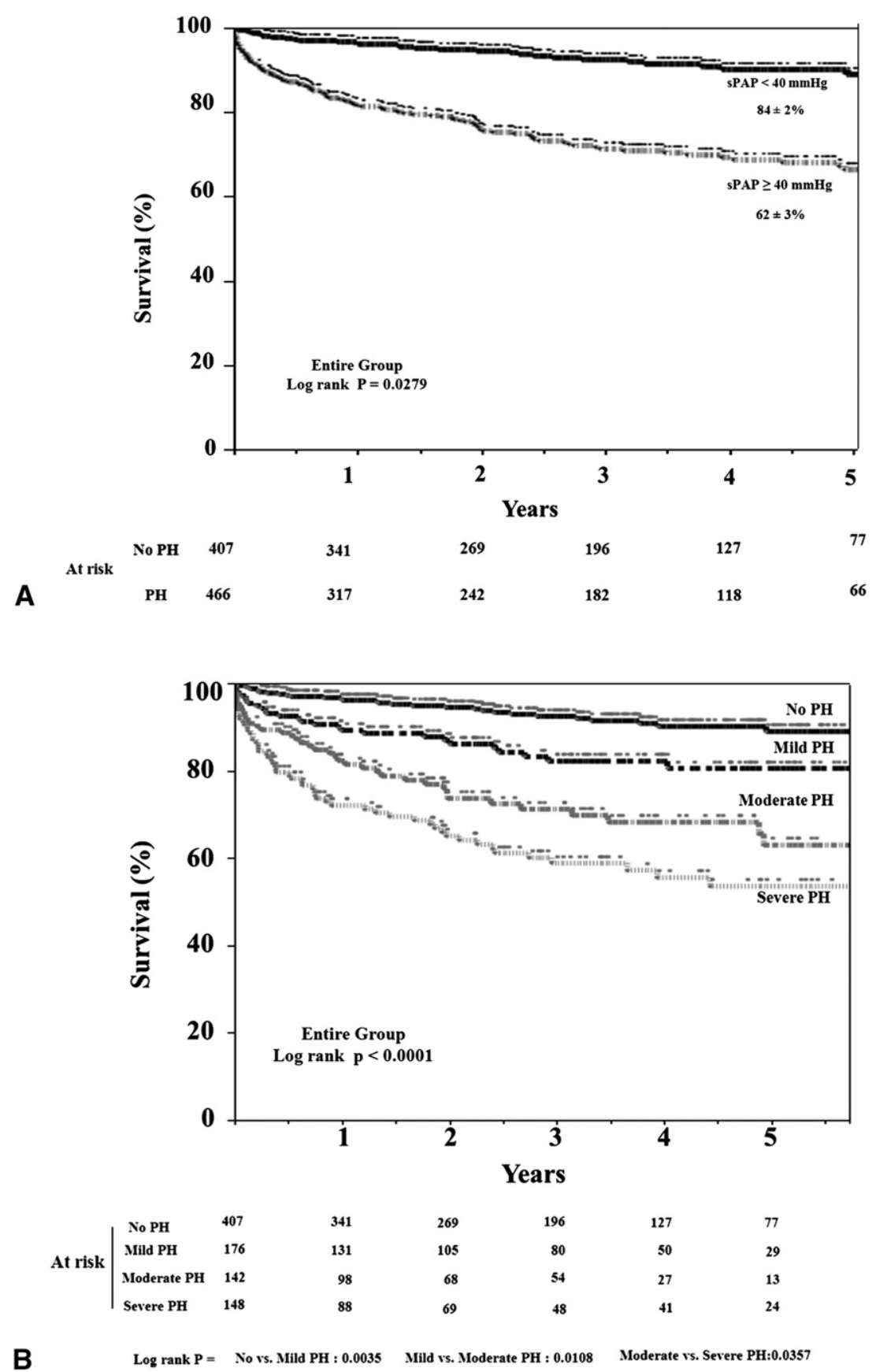

FIGURE 3. Long-term survival according to preoperative PH. A, Actuarial survival according to a cutoff value of sPAP $40 \mathrm{~mm} \mathrm{Hg}$ or greater. B, Survival according to preoperative $\mathrm{PH}$ grade. $P H$, Pulmonary hypertension; $s P A P$, Systolic pulmonary artery pressure.

to preoperative SPAP. Pulmonary artery pressures were measured in the majority of patients with RHC. On the basis of the cutoff point of preoperative SPAP $40 \mathrm{~mm} \mathrm{Hg}$ or greater, $\mathrm{PH}$ was observed in more than half of the population. Compared with patients with no $\mathrm{PH}$ (sPAP $<40 \mathrm{~mm}$ $\mathrm{Hg})$, patients presenting with mild $\mathrm{PH}(40 \leq \mathrm{sPAP}<50$ $\mathrm{mm} \mathrm{Hg}$ ) had lower left ventricular ejection fractions, more atrial fibrillation and more tricuspid regurgitation. Operative mortality among patients with no $\mathrm{PH}$
(sPAP $<40 \mathrm{~mm} \mathrm{Hg}$ ) was low $(2 \%)$. With increased sPAP $40 \mathrm{~mm} \mathrm{Hg}$ or greater or sPAP/sBP 0.3 or greater, higher mortality and morbidity were observed after surgery. Long-term survival was compromised by the presence of any degree of preoperative $\mathrm{PH}$, including those patients with mild $\mathrm{PH}(40 \leq \mathrm{sPAP}<50 \mathrm{~mm} \mathrm{Hg})$. These findings were also evident in patients with isolated degenerative MR. Pulmonary artery pressures declined immediately after operation but not thereafter, and residual $\mathrm{PH}$ 
TABLE 6. Predictors of long-term mortality*

\begin{tabular}{lccc}
\hline \multicolumn{1}{c}{ Variable } & HR & $\mathbf{9 5} \%$ CI & \multicolumn{1}{c}{$\boldsymbol{P}$} \\
\hline sPAP (per 1 mm Hg increment) & 1.018 & $1.007-1.028$ & .001 \\
NYHA function class (III /IV vs I/II) & 1.835 & $1.216-2.844$ & .0034 \\
Age (per unit) & 1.036 & $1.021-1.051$ & $<.0001$ \\
Dialysis (yes vs no) & 3.052 & $1.596-5.452$ & .0013 \\
Diabetes (yes vs no) & 1.826 & $1.222-2.688$ & .0037 \\
\hline$C I$, Confidence interval; $H R$, hazard ratio; $N Y H A$, New York Heart Association; $s P A P$, & \\
systolic pulmonary artery pressure. ${ }^{*} \mathrm{C}$-statistic of multivariable model: 0.764.
\end{tabular}

was observed late among all patients except those with normal preoperative $\mathrm{SPAP}(\mathrm{s})$.

The prevalence of $\mathrm{PH}$ in patients undergoing surgery for MR depends on the threshold of sPAP used to define PH. Although the cutoff value of sPAP to define PH (sPAP $>50$ $\mathrm{mm} \mathrm{Hg}$ ) in current guidelines is derived from small clinical series performed on patients with systemic sclerosis, ${ }^{18}$ various values are frequently used in practice. ${ }^{8,19,20}$ We used a lower threshold of sPAP value (sPAP $\geq 40 \mathrm{~mm} \mathrm{Hg}$ ) compared with existing guidelines to identify individuals at risk for adverse outcomes after surgery. Outcomes after surgery for patients with no PH (sPAP $<40 \mathrm{~mm} \mathrm{Hg}$ ) were excellent. Operative mortality was $2 \%$, and $86 \%$ survived more than 5 years after surgery. However, approximately 1 of 10 patients with severe $\mathrm{PH}(\mathrm{sPAP} \geq 60 \mathrm{~mm} \mathrm{Hg})$ undergoing surgery for MR died in hospital or early after surgery, and only half survived 5 years after surgery. Long-term survival in patients with isolated degenerative MR was compromised among patients with any degree of preoperative $\mathrm{PH}$.

In both cohorts in this study, preoperative sPAP was an important independent predictor for long-term survival after surgery. Even the presence of mild $\mathrm{PH}(40 \leq$ sPAP $<50 \mathrm{~mm} \mathrm{Hg}$ ) compromised long-term survival in both cohorts. These results support a management strategy of surgical intervention for MR before the sPAP reaches 50 mm Hg. Le Tourneau and colleagues ${ }^{2}$ studied 256 patients with organic MR referred for MV surgery. Preoperative $\mathrm{PH}$ ( $\mathrm{sPAP} \geq 50 \mathrm{~mm} \mathrm{Hg}$ ) was present in 83 patients. Although PH did not significantly increase operative mortality, survival 8 years after surgery was $86 \%$ in patients with no $\mathrm{PH}$ compared with $58 \%$ in those with sPAP $50 \mathrm{~mm} \mathrm{Hg}$ or greater. Patients with preoperative $\mathrm{PH}$ were more symptomatic early after surgery and had more left ventricular dysfunction compared with those with no PH. Le Tourneau and colleagues ${ }^{2}$ found that long-term risk for mortality increased by $50 \%$ per $10 \mathrm{~mm} \mathrm{Hg}$ increase in preoperative sPAP. $^{2}$ Barbieri and colleagues ${ }^{7}$ studied 437 patients with degenerative severe MR with flail leaflet. All patients had no or minimal symptoms with normal left ventricular

TABLE 7. Baseline characteristics of the patients with isolated leaflet prolapse $(n=284)$ according to the degree of preoperative pulmonary hypertension*

\begin{tabular}{|c|c|c|c|c|}
\hline Variable & $\begin{array}{c}\text { sPAP }<40 \mathrm{~mm} \text { Hg } \\
193(68 \%)\end{array}$ & $\begin{array}{c}40 \leq \text { sPAP }<50 \text { mm Hg } \\
48(17 \%)\end{array}$ & $\begin{array}{c}50 \mathrm{~mm} \mathrm{Hg} \leq \text { sPAP } \\
43(15 \%)\end{array}$ & $P$ value \\
\hline \multicolumn{5}{|l|}{ sPAP (mm Hg) } \\
\hline Mean \pm SD & $28 \pm 6$ & $44 \pm 3$ & $63 \pm 13$ & $<.0001$ \\
\hline Median (25th-75th percentile) & $29(25-33)$ & $44(40-46)$ & $59(51-70)$ & \\
\hline Age (y) & $55 \pm 12$ & $65 \pm 14$ & $62 \pm 13$ & .001 \\
\hline Female gender & $60(31 \%)$ & $19(40 \%)$ & $22(51 \%)$ & .0370 \\
\hline $\mathrm{BMI}\left(\mathrm{kg} / \mathrm{m}^{2}\right.$, mean $\left.\pm \mathrm{SD}\right)$ & $26 \pm 6$ & $27 \pm 6$ & $30 \pm 8$ & .0045 \\
\hline Morbid obesity $\dagger$ & $5(39 \%)$ & $2(15 \%)$ & $6(46 \%)$ & .445 \\
\hline NYHA class III/IV & $57(30 \%)$ & $27(56 \%)$ & $27(63 \%)$ & $<.0001$ \\
\hline Atrial fibrillation & $38(20 \%)$ & $19(40 \%)$ & $13(30 \%)$ & .0109 \\
\hline LVEF (\%) & $58 \pm 8$ & $56 \pm 8$ & $55 \pm 11$ & .0414 \\
\hline LVEDD (cm) & $5.5 \pm 0.7$ & $5.6 \pm 0.7$ & $5.6 \pm 0.8$ & .5551 \\
\hline $\operatorname{LVESD}(\mathrm{cm})$ & $3.4 \pm 0.7$ & $3.7 \pm 0.8$ & $3.8 \pm 0.8$ & .0088 \\
\hline LA diameter $(\mathrm{cm})$ & $4.5 \pm 0.7$ & $4.8 \pm 0.7$ & $4.9 \pm 0.7$ & .0020 \\
\hline RV dysfunction & $3(2 \%)$ & $3(6 \%)$ & $7(16 \%)$ & .0001 \\
\hline MR grade & $3.7 \pm 0.5$ & $3.4 \pm 0.7$ & $3.6 \pm 0.5$ & .0207 \\
\hline TR grade & $0.4 \pm 0.6$ & $1.0 \pm 1.0$ & $1.4 \pm 1.1$ & $<.0001$ \\
\hline Chronic lung disease & $0(0 \%)$ & $0(0 \%)$ & $0(0 \%)$ & - \\
\hline Diabetes mellitus & $5(3 \%)$ & $4(8 \%)$ & $7(16 \%)$ & .0013 \\
\hline Hypertension & $88(46 \%)$ & $30(63 \%)$ & $27(63 \%)$ & .0275 \\
\hline History of stroke & $2(1 \%)$ & $0(0 \%)$ & $3(7 \%)$ & .0149 \\
\hline Previous MI & $1(1 \%)$ & $2(4 \%)$ & $1(2 \%)$ & .1359 \\
\hline Renal failure (dialysis) & $0(0 \%)$ & $0(0 \%)$ & $1(2 \%)$ & .0601 \\
\hline
\end{tabular}

Data presented in mean \pm SD or n (\%). BMI, Body mass index; $L A$, left atrium; $L V E F$, left ventricular ejection fraction; $L V E D D$, left ventricular end-diastolic diameter; $L V E S D$, left ventricular end-diastolic diameter; $M I$, myocardial infarction; $M R$, mitral regurgitation; $N Y H A$, New York Heart Association; $R V$, right ventricle; $s P A P$, systolic pulmonary artery pressure; $T R$, tricuspid regurgitation. ${ }^{*} \mathrm{PH}$ absent: $\mathrm{sPAP}<40 \mathrm{~mm} \mathrm{Hg}$; mild $\mathrm{PH}: 40 \leq \mathrm{sPAP}<50 \mathrm{~mm} \mathrm{Hg}$; moderate $\mathrm{PH}: 50 \leq \mathrm{sPAP}<60 \mathrm{~mm} \mathrm{Hg}$; Severe PH: sPAP $\geq 60 \mathrm{~mm} \mathrm{Hg}$. $\dagger$ Morbid obesity: BMI $\geq 40 \mathrm{~kg} / \mathrm{m}^{2}$. 


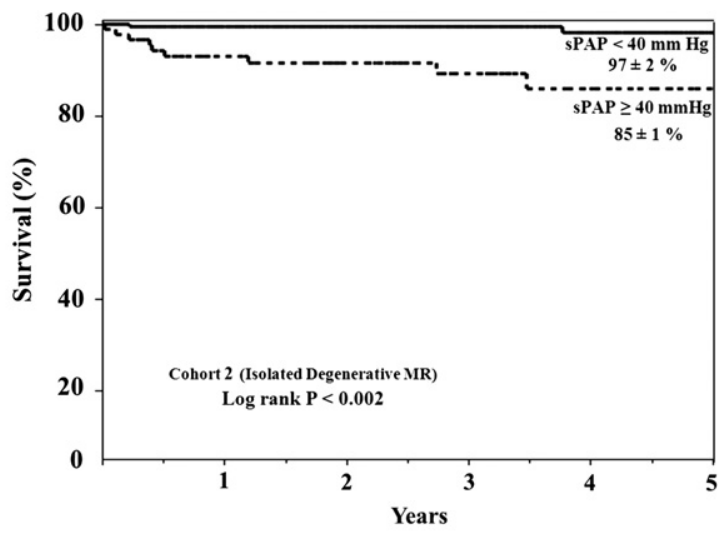

A

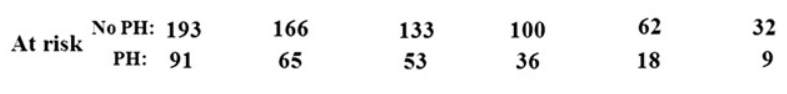

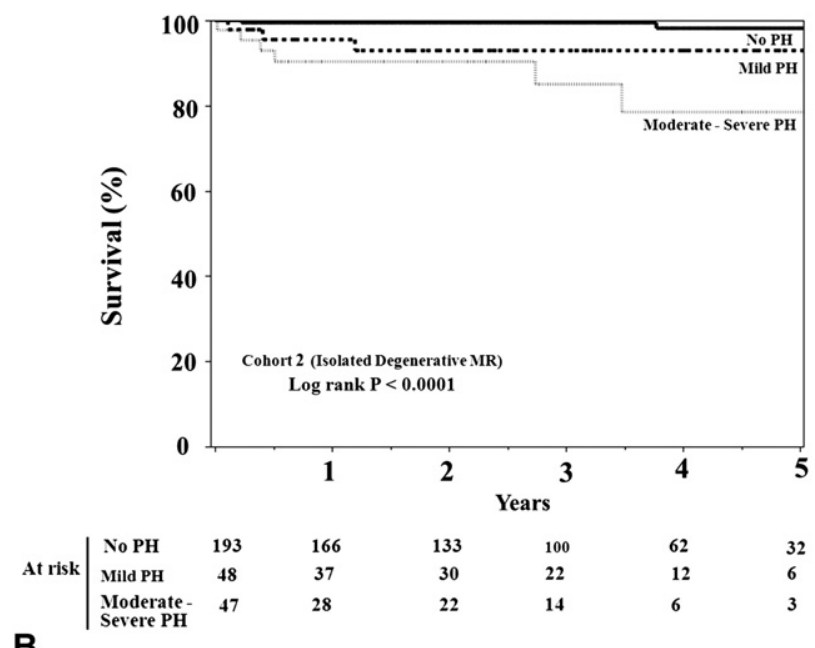

B

FIGURE 4. Survival curve according to preoperative $\mathrm{PH}$ (sPAP $\geq 40 \mathrm{~mm}$ $\mathrm{Hg}$ ) in cohort 2 (patients with isolated degenerative MR due to flail leaflet). For all patients (A) and according to preoperative $\mathrm{PH}$ grade (B). MR, Mitral regurgitation; $P H$, pulmonary hypertension; $s P A P$, systolic pulmonary artery pressure.

function. Baseline PH (sPAP $>50 \mathrm{~mm} \mathrm{Hg}$ ) was present in $23 \%$ of patients. Actuarial survival 5 years after surgery was $86 \%$ in patients with no $\mathrm{PH}(\mathrm{sPAP}<50 \mathrm{~mm} \mathrm{Hg}$ ) compared with $63 \%$ in those with $\mathrm{PH}(\mathrm{sPAP}>50 \mathrm{~mm} \mathrm{Hg}$ ). MV surgery was eventually performed in $75 \%$ of patients. Postoperative survival was significantly decreased in patients with baseline sPAP $50 \mathrm{~mm} \mathrm{Hg}$ or greater. ${ }^{7}$ The authors concluded that $\mathrm{PH}(\mathrm{sPAP}>50 \mathrm{~mm} \mathrm{Hg}$ ) in patients with chronic organic MR carries an increased risk of long-term mortality, which is similar to our experience. ${ }^{2}$ They found that MV surgery is beneficial to improve sPAP, but surgery cannot completely abolish adverse effects of $\mathrm{PH}$ on outcomes. ${ }^{7}$

Studies have shown that sustained systemic hypertension correlates with higher values of pulmonary artery pressures ${ }^{21}$ and suggested that interpretation of sPAP in hypertensive patients should take into account $\mathrm{SBP}$ value. ${ }^{21} \mathrm{We}$ studied the pulmonary artery to systemic blood pressure in our population and found nearly identical results to our initial analysis that examined only sPAP. We found that elevated $\mathrm{sPAP} / \mathrm{sBP}$ ratio (sPAP/sBP $\geq 0.3$ ) compromised both early and late survival after MV surgery for MR.

\section{No Pulmonary Hypertension Versus Mild Pulmonary Hypertension: Lessons Learned}

Operative mortality and major morbidity were similar between patients with no $\mathrm{PH}$ (sPAP $<40 \mathrm{~mm} \mathrm{Hg}$ ) and those with mild $\mathrm{PH}(40 \leq \mathrm{sPAP}<50 \mathrm{~mm} \mathrm{Hg})$. However, longterm survival was lower in patients with mild PH compared with patients with no preoperative $\mathrm{PH}$ in the overall study population, in the cohort with isolated leaflet prolapse, and in patients with no or minimal preoperative symptoms. Given the increased risk of adverse outcomes in patients with mild $\mathrm{PH}(40 \leq \mathrm{sPAP}<50 \mathrm{~mm} \mathrm{Hg})$ compared with those with no PH (sPAP $<40 \mathrm{~mm} \mathrm{Hg}$ ), we believe that referral for MV surgery for MR should be considered before sPAP exceeds $40 \mathrm{~mm} \mathrm{Hg}$.

\section{Regression of Pulmonary Artery Pressures After Mitral Valve Surgery}

In this study, significant reduction in mean SPAP was observed early after operation. During follow-up, the mean SPAP failed to return to normal levels in patients with any degree of preoperative $\mathrm{PH}$ ( $\mathrm{sPAP} \geq 40 \mathrm{~mm} \mathrm{Hg}$ ). Incomplete improvement in pulmonary artery pressures after operation for MR suggests that significant pulmonary vascular disease develops early in the presence of MR, and therefore a management strategy that triggers operative intervention when the sPAP exceeds $40 \mathrm{~mm} \mathrm{Hg}$ will improve long-term survival.

\section{Study Limitations}

The present study was subject to the limitations inherent in a retrospective study. We did not exclude patients with aortic valve disease or chronic obstructive pulmonary disease in the first cohort. Although we performed multivariable analysis to account for confounding factors, we cannot guarantee that unmeasured confounding factors did not influence our results. Preoperative evaluation of pulmonary pressures was comprehensive, although predischarge and follow-up echocardiographic data were incomplete. We did not have data to evaluate the impact of residual $\mathrm{PH}$ on other outcomes, such as freedom from heart failure, atrial fibrillation, or NYHA function class at follow-up. Medical therapy after surgery was not assessed and might affect long-term outcomes.

\section{CONCLUSIONS}

PH is a common adverse sequela of MR. Systolic pulmonary artery pressure is a predictor of operative mortality, morbidity, and long-term death. Even mild PH ( $40 \leq$ sPAP $<50 \mathrm{~mm} \mathrm{Hg}$ ) compromises outcomes after MV 


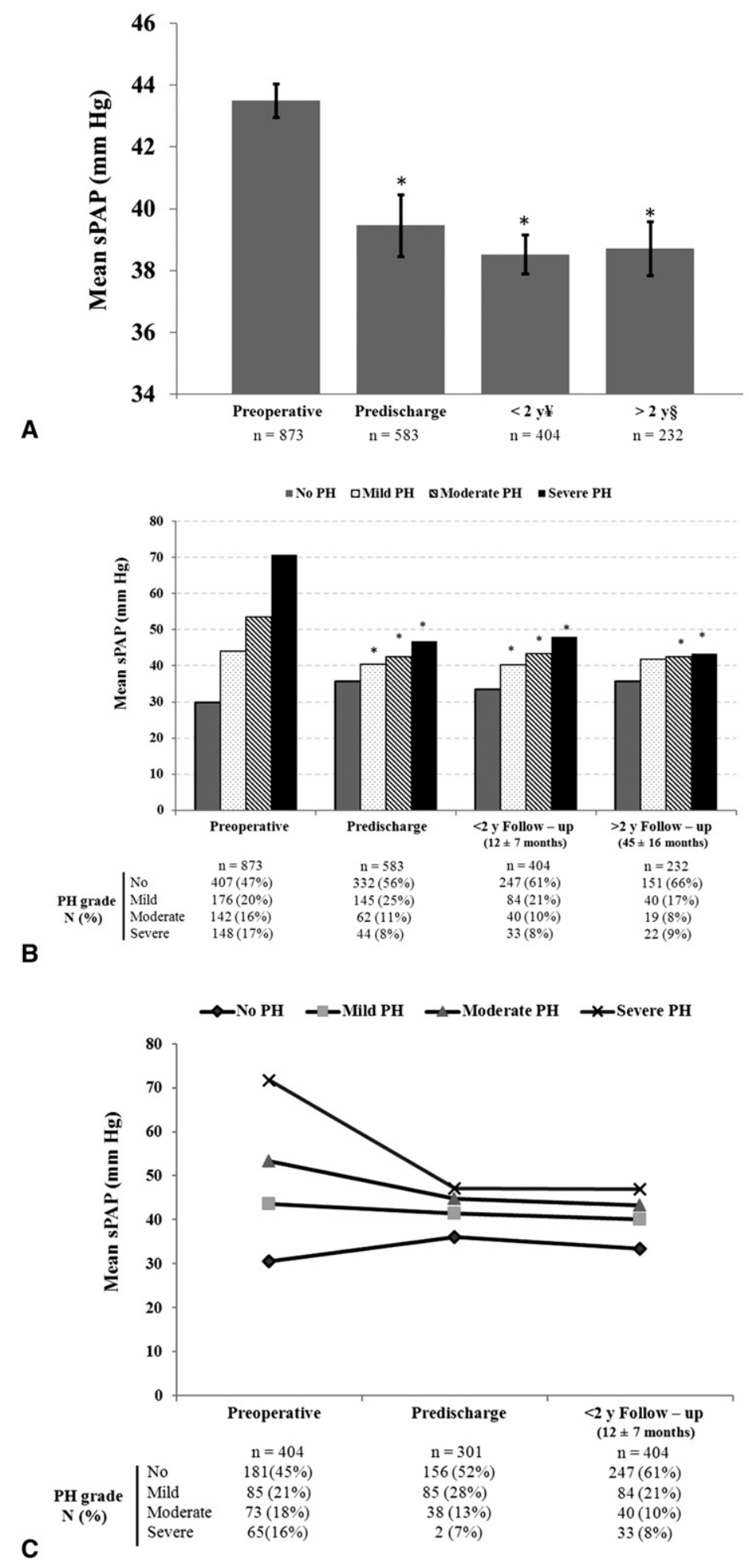

FIGURE 5. Mean SPAP at different time points. A, All patients (mean \pm standard deviation sPAP at preoperative: $43 \pm 16$, predischarge: $39 \pm 11$, followup $<2$ years: $39 \pm 12$, follow-up $>2$ years: $38 \pm 13 \mathrm{~mm} \mathrm{Hg}$ ). B, According to PH grade for all patients. C, For 404 patients with 2-year follow-up. Mean sPAP decreased markedly in patients with higher preoperative sPAP, but residual sPAP $40 \mathrm{~mm} \mathrm{Hg}$ or greater was more common after surgery and during follow-up in these groups. Pulmonary pressures at predischarge relatively correlate with the late response of pulmonary pressure changes to surgery (no significant difference was identified in mean sPAP at predischarge and within 2 years follow-up, $P=.95$ ). ¥Median: 12 months (24 days to 24 months). $\S$ Median: 41 months (24-91 months). $* P<.01$ compared with preoperative value. $M R$, Mitral regurgitation; $P H$, pulmonary hypertension; $s P A P$, Systolic pulmonary artery pressure. 
surgery for MR. sPAP improved early after surgery, although residual PH was commonly observed among all patients except those with normal preoperative $\operatorname{SPAP}(<40 \mathrm{~mm}$ $\mathrm{Hg}$ ). Referral for MV surgery for MR should be considered in the presence of an SPAP $40 \mathrm{~mm} \mathrm{Hg}$ or greater.

\section{References}

1. Montant P, Chenot F, Robert A, Vancraeynest D, Pasquet A, Gerber B, et al. Long-term survival in asymptomatic patients with severe degenerative mitral regurgitation: a propensity score-based comparison between an early surgical strategy and a conservative treatment approach. J Thorac Cardiovasc Surg. 2009;138: 1339-48.

2. Le Tourneau T, Richardson M, Juthier F, Modine T, Fayad G, Polge A-S, et al. Echocardiography predictors and prognostic value of pulmonary artery systolic pressure in chronic organic mitral regurgitation. Heart. 2010;96:1311-7.

3. Alexopoulos D, Lazzam C, Borrico S, Fiedler L, Ambrose JA. Isolated chronic mitral regurgitation with preserved systolic left ventricular function and severe pulmonary hypertension. J Am Coll Cardiol. 1989;14:319-22.

4. Cesnjevar RA, Feyrer R, Walther F, Mahmoud FO, Lindemann Y, von der Emde J. High-risk mitral valve replacement in severe pulmonary hypertension-30 years experience. Eur J Cardiothorac Surg. 1998;13:344-52.

5. Grigioni F, Branzi A. Management of asymptomatic mitral regurgitation. Heart. 2010;96:1938-45.

6. Topilsky Y, Suri R, Schaff HV. Enriquez-Sarano M. When to intervene for asymptomatic mitral valve regurgitation. Semin Thorac Cardiovasc Surg. 2010;22:216-24.

7. Barbieri A, Bursi F, Grigioni F, Tribouilloy C, Avierinos JF, Michelena HI, et al. Prognostic and therapeutic implications of pulmonary hypertension complicating degenerative mitral regurgitation due to flail leaflet: a multicenter longterm international study. Eur Heart J. 2010;32:751-9.

8. Parker MW, Mittleman MA, Waksmonski CA, Sanders G, Riley MF, Douglas PS, et al. Pulmonary hypertension and long-term mortality in aortic and mitral regurgitation. Am J Med. 2010;123:1043-8.

9. Kang D-H, Kim JH, Rim JH, Kim M-J, Yun S-C, Song J-M, et al. Comparison of early surgery versus conventional treatment in asymptomatic severe mitral regurgitation. Circulation. 2009;119:797-804.

10. Bonow RO, Carabello BA, Chatterjee K, de Leon AC Jr, Faxon DP, Freed MD, et al. ACC/AHA 2006 Guidelines for the Management of Patients With Valvular Heart Disease: A Report of the American College of Cardiology/American Heart Association Task Force on Practice Guidelines (Writing Committee to Revise the 1998 Guidelines for the Management of Patients With Valvular Heart Disease): developed in collaboration with the Society of Cardiovascular Anesthesiologists: endorsed by the Society for Cardiovascular Angiography and Interventions and the Society of Thoracic Surgeons. Circulation. 2006;114:e84-231.

11. Vahanian A, Baumgartner H, Bax J, Butchart E, Dion R, Filippatos G, et al. Guidelines on the management of valvular heart disease. Eur Heart J. 2007; 28:230-68.

12. O'Brien SM, Shahian DM, Filardo G, Ferraris VA, Haan CK, Rich JB, et al. The Society of Thoracic Surgeons 2008 cardiac surgery risk models: part 2-isolated valve surgery. Ann Thorac Surg. 2009;88(1 Suppl):S23-42.

13. Enriquez-Sarano M, Akins CW, Vahanian A. Mitral regurgitation. Lancet. 2009; 373:1382-94

14. Kircher BJ, Himelman RB, Schiller NB. Noninvasive estimation of right atrial pressure from the inspiratory collapse of the inferior vena cava. Am J Cardiol. 1990;66:493-6.

15. Zoghbi WA, Enriquez-Sarano M, Foster E, Grayburn PA, Kraft CD, Levine RA, et al. Recommendations for evaluation of the severity of native valvular regurgitation with two-dimensional and Doppler echocardiography. $J$ Am Soc Echocardiogr. 2003;16:777-802.

16. Carpentier A. Cardiac valve surgery - the "French correction" J Thorac Cardiovasc Surg. 1983;86:323-37.

17. Cowper DC, Kubal JD, Maynard C, Hynes DM. A Primer and comparative review of major U.S. mortality databases. Ann Epidemiol. 2002;12:462-8.

18. Galiè N, Hoeper MM, Humbert M, Torbicki A, Vachiery J-L, et al., Authors/Task Force M. Guidelines for the diagnosis and treatment of pulmonary hypertension. Eur Heart J. 2009;30:2493-537.

19. Lam CSP, Roger VL, Rodeheffer RJ, Borlaug BA, Enders FT, Redfield MM. Pulmonary hypertension in heart failure with preserved ejection fraction: a community-based study. J Am Coll Cardiol. 2009;53:1119-26.
20. Walls M, Cimino N, Bolling S, Bach D. Persistent pulmonary hypertension after mitral valve surgery: does surgical procedure affect outcome? J Heart Valve Dis 2008; 17:1-9.

21. Abergel E, Chtellier G, Toussaint P, Dib JC, Menard J, Diebold B. Dopplerderived pulmonary arterial systolic pressure in patients with known systemic arterial pressures. Am J Cardiol. 1996;77:767-9.

\section{APPENDIX 1. VARIABLES USED IN ANALYSIS}

Demographics: age (y), gender, sex, weight $(\mathrm{kg})$, height $(\mathrm{cm})$, body mass index $\left(\mathrm{kg} / \mathrm{m}^{2}\right)$, NYHA function class (I-IV), emergency operation.

Cardiac stricture and function: left ventricular ejection fraction $(\%)$, sPAP ( $\mathrm{mm} \mathrm{Hg})$, left ventricular inner systolic diameter $(\mathrm{cm})$, left ventricular inner diastolic diameter $(\mathrm{cm})$, LA diameter $(\mathrm{cm})$, right ventricular dysfunction $(0=$ no, $1=$ yes $)$, MV regurgitation, aortic valve regurgitation, aortic valve stenosis, tricuspid valve regurgitation.

Cardiac comorbidity: number of coronary vessels with $>50 \%$ stenosis, left main coronary disease $\geq 50 \%$ stenosis, atrial fibrillation. ventricular arrhythmia, family history of coronary artery disease, dyslipidemia, heart failure, hypertension, preoperative pacemaker, previous myocardial infarction, endocarditis.

Noncardiac morbidity: diabetes, chronic obstructive pulmonary disease, peripheral arterial disease, dialysis, stroke, smoking.

Procedures: MV repair/replacement, tricuspid valve surgery, aortic valve repair/replacement, coronary artery bypass grafting, surgical ablation for atrial fibrillation, reoperation.

\section{APPENDIX 2. ANALYSIS OF OUTCOMES FOR ISOLATED MITRAL VALVE OPERATION FOR MITRAL REGURGITATION}

Patients with previous myocardial infarction $(\mathrm{n}=138)$, concomitant aortic valve surgery $(\mathrm{n}=100)$, and coronary artery bypass surgery $(n=253)$ were excluded, leaving

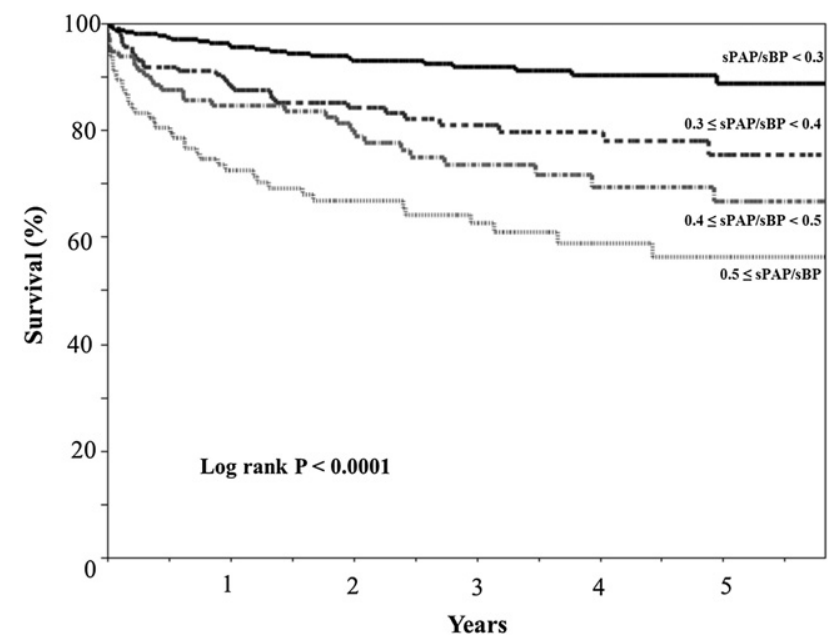

APPENDIX FIGURE 1. Kaplan-Meier survival curve according to preoperative sPAP/sBP. $s P A P$, Systolic pulmonary artery pressure; $s B P$, systolic blood pressure. 


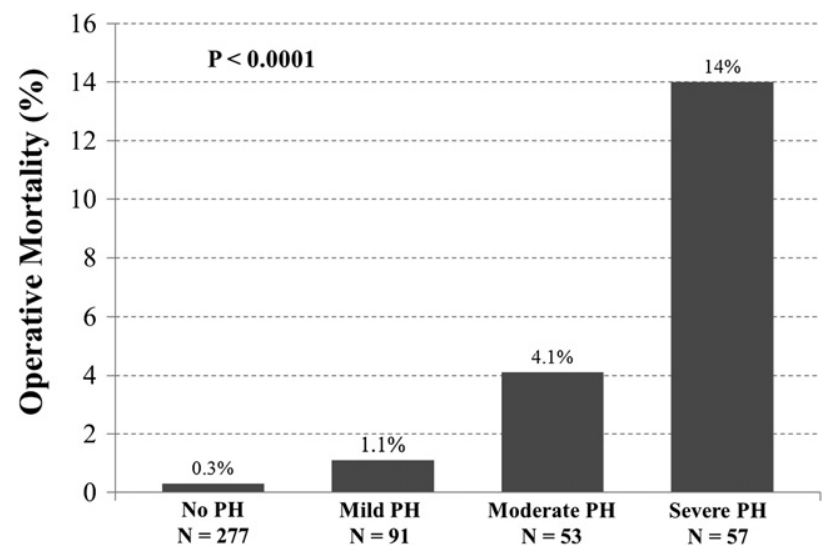

APPENDIX FIGURE 2. Hospital mortality according to preoperative PH grade among patients who underwent isolated MV operation for MR $(\mathrm{n}=478) . P H$, Pulmonary hypertension.

478 patients for analysis. Patients were categorized as having no $\mathrm{PH}(\mathrm{sPAP}<40 \mathrm{~mm} \mathrm{Hg})(\mathrm{n}=277,58 \%)$, mild $\mathrm{PH}$ $(40 \leq$ sPAP $<50 \mathrm{~mm} \mathrm{Hg})(\mathrm{n}=91,19 \%)$, moderate $\mathrm{PH}$ $(50 \leq \operatorname{sPAP}<60 \mathrm{~mm} \mathrm{Hg})(\mathrm{n}=53,11 \%)$, or severe $\mathrm{PH}$ $(\mathrm{sPAP} \geq 60 \mathrm{~mm} \mathrm{Hg})(\mathrm{n}=57,12 \%)$. Overall operative mortality was $2.3 \%(n=11)$. Operative mortality (Appendix

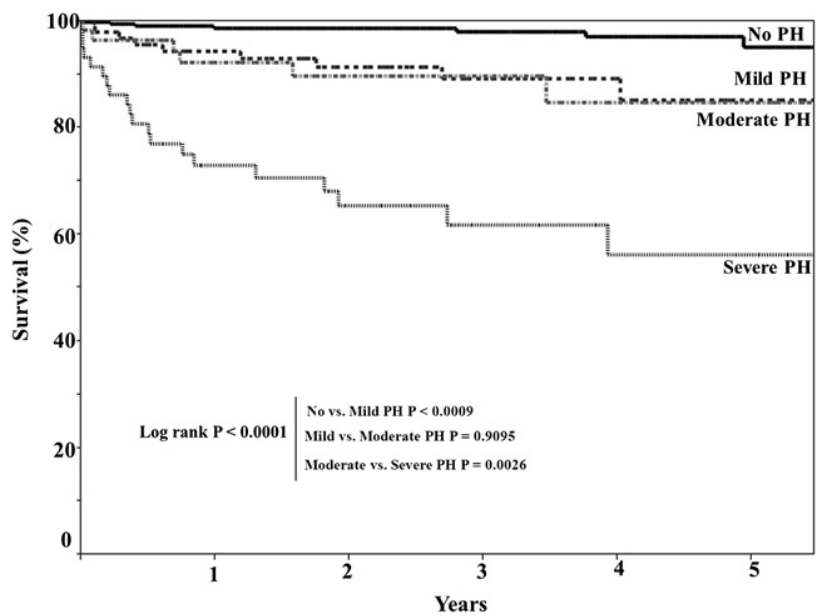

APPENDIX FIGURE 3. Long-term survival according to preoperative $\mathrm{PH}$ among patients who underwent isolated MV operation for MR $(\mathrm{n}=478) . P H$, Pulmonary hypertension.

Figure 2) and long-term death (Appendix Figure 3) were significantly higher among patients with higher preoperative sPAP. Preoperative sPAP (per 1-mm increase) was an independent predictor for both operative mortality and long-term death (Appendix Table 1).

APPENDIX TABLE 1. Predictors of early and late mortality for 478 patients who underwent isolated mitral valve operation for mitral regurgitation

\begin{tabular}{|c|c|c|c|c|c|c|}
\hline \multirow[b]{2}{*}{ Predictor } & \multicolumn{3}{|c|}{ Univariate analysis } & \multicolumn{3}{|c|}{ Multivariate analysis } \\
\hline & OR & $95 \%$ CI & $P$ & OR & $95 \%$ CI & $P$ \\
\hline \multicolumn{7}{|l|}{ Early mortality } \\
\hline sPAP (per 1-mm increase) & 1.063 & $1.0319-1.0981$ & .0001 & 1.0585 & $1.013-1.087$ & .0018 \\
\hline NYHA (III/IV vs I/II) & 4.923 & $1.252-35.57$ & .0207 & & & \\
\hline TV surgery & 4.280 & $1.0929-14.923$ & .0381 & & & \\
\hline Dialysis & 19.955 & $4.761-76.92$ & .0002 & 10.34 & $2.347-41.66$ & .0033 \\
\hline History of stroke & 6.099 & $1.545-21.141$ & .0124 & & & \\
\hline Late mortality & HR & & & HR & & \\
\hline sPAP (per 1-mm increase) & 1.0533 & $1.0374-1.0687$ & $<.0001$ & 1.0387 & $1.021-1.056$ & $<.0001$ \\
\hline Gender (male vs female) & 2.0256 & $1.1133-3.7900$ & .0206 & & & \\
\hline NYHA (III/IV vs I/II) & 4.3928 & $2.2036-9.7415$ & $<.0001$ & 2.46 & $1.193-5.635$ & .0138 \\
\hline Age (per year) & 1.0291 & $1.0072-1.05179$ & .0086 & 1.059 & $1.0327-1.0877$ & $<.0001$ \\
\hline LV ejection fraction & 1.0345 & $1.0105-1.0563$ & .0059 & 1.028 & $1.002-1.0537$ & .0373 \\
\hline LA dimension $(\mathrm{cm})$ & 1.0459 & $1.0059-1.0855$ & .0242 & & & \\
\hline TR grade & 1.6174 & $1.3124-1.9701$ & $<.0001$ & 1.470 & $1.152-1.859$ & .0023 \\
\hline MV replacement & 3.2775 & $1.5792-6.2936$ & .0023 & & & \\
\hline TV surgery & 2.6871 & $1.2953-5.1547$ & .0096 & & & \\
\hline RV dysfunction & 3.3092 & $1.4909-6.6058$ & .0048 & & & \\
\hline Diabetes & 3.0498 & $1.50663-5.7724$ & .0028 & & & \\
\hline Hypertension & 2.255 & $1.206-4.465$ & .0102 & & & \\
\hline Infective endocarditis & 3.3201 & $1.7900-6.0237$ & .0002 & 9.651 & $4.127-22.901$ & $<.0001$ \\
\hline Chronic lung disease & 3.1836 & $14897-6.2100$ & .0041 & 2.280 & $1.051-4.544$ & .0379 \\
\hline Peripheral arterial disease & 4.1319 & $1.2934-10.7091$ & .0211 & & & \\
\hline History of stroke & 3.6746 & $1.809-6.979$ & .0006 & & & \\
\hline Atrial fibrillation & 2.0265 & $1.0936-3.6734$ & .0255 & & & \\
\hline Emergency operation & 6.099 & $1.545-21.141$ & .0124 & & & \\
\hline
\end{tabular}

$C I$, Confidence interval; $L A$, left atrial; $L V$, left ventricle; $M V$, mitral valve; $N Y H A$, New York Heart Association; $O R$, odds ratio; $R V$, right ventricle; $s P A P$, systolic pulmonary artery pressure; $T R$, tricuspid regurgitation; $T V$, tricuspid valve. 


\section{Discussion}

Dr Gerald Lawrie (Houston, Tex). This is a large series of patients, the biggest reported to date, and it also includes the broader spectrum of severity of disease of any previous report. It is also unique in that many of the patients had their PH diagnosed by RHC. Up to $40 \%$ of patients in previous studies have been excluded because insufficient tricuspid regurgitation has been present to allow them to assess pulmonary artery pressure by echocardiography.

This study has confirmed the findings of smaller earlier studies that an SPAP greater than $50 \mathrm{~mm}$ is a strong independent predictor of early and late survival. This study also demonstrated that in all categories of $\mathrm{PH}$, regression of $\mathrm{PH}$ is incomplete after surgery, reaching approximately $40 \mathrm{~mm}$ systolic after 2 years. Despite the fact that surgery improves the prognosis at all levels of pulmonary artery pressure, regression is incomplete because the $\mathrm{PH}$ seen in severe MR arises not only from the MR itself but also from a series of complex changes in the left ventricle and LA morphology and their systolic and diastolic function. The pulmonary artery bed may develop increased vascular tone, and some degree of pulmonary hypertensive vasculopathy may develop as seen in mitral stenosis. Some of these changes, once present, may not regress despite relief of the MR.

These findings and those of this study suggest it may be important to pursue the issue of PH more aggressively and specifically than we have done to date.

First, these data provide further strong support for surgery early after the diagnosis of asymptomatic severe MR. Intraoperatively, improvement of LA function by atrial fibrillation ablation and volume reduction surgery could benefit, and I think we need to look more carefully at the influence of surgery on left ventricular diastolic function to try to refine our techniques to preserve left ventricular and LA function.

In patients presenting with $\mathrm{PH}$ in the 60 to $80 \mathrm{~mm}$ range, we have for many years involved our pulmonologists early preoperatively to evaluate and manage the $\mathrm{PH}$ and exclude other contributory causes. It is noteworthy that $21 \%$ of the patients in this study with pulmonary artery pressures greater than 60 had significant chronic obstructive pulmonary disease. It may be that this pulmonary evaluation should be extended to include RHC in all cases with drug evaluation for pulmonary artery vascular responsiveness.

Because incomplete regression of the pulmonary artery pressure was still present 2 years after surgery, it also may be that these patients should be followed long-term by a pulmonologist, something we have not done, and a cardiologist. Long-term pulmonary artery drug therapy could be beneficial in some cases.

Thank you for this excellent presentation, which I think has brought a neglected topic to our attention. I do have 2 questions for the presenter.

What proportion of the patients with no tricuspid regurgitation on echo had RHC showing significant PH? Do you think that all patients undergoing mitral repair with no TR should have RHC to assess their pulmonary artery pressure before they go to surgery? The second question relates to the fact that you agree we have a big problem here that we have neglected and that the approach to the care of these patients with persistent significant $\mathrm{PH}$ should involve long-term follow-up with a pulmonologist with active treatment to moderate their $\mathrm{PH}$.
Dr Ghoreishi. To respond to your first question, we could not assess the sPAP based on echocardiography because of an absent tricuspid regurgitation jet velocity in $15 \%$ of patients. Among these patients, sPAPs were greater than 40 in $37 \%$ as measured on RHC. In 373 patients who were studied with both echocardiography and RHC, we found a reasonably good correlation between the results of echo and RHC, although there was significant variability. We expect some discrepancies between measurement of sPAP with RHC and echocardiography. Pulmonary artery pressures are dynamic, and the echocardiography and RHC were performed at different times.

We believe that the current recommendation to operate after the sPAP exceeds $50 \mathrm{~mm} \mathrm{Hg}$ is associated with compromised early and late outcomes, and therefore we are recommending moving that threshold back to $40 \mathrm{~mm} \mathrm{Hg}$.

We advocate RHC for patients with asymptomatic severe MR who are managed nonsurgically to make sure that PH (sPAP $\geq$ $40 \mathrm{~mm} \mathrm{Hg}$ ) is not present.

To respond to your second question, we currently do not have any therapeutic protocols directed at residual $\mathrm{PH}$. We found that during follow-up, sPAP in patients with preoperative PH (sPAP $\geq 40 \mathrm{~mm} \mathrm{Hg}$ ) never decreased to normal after surgery. We agree with you that these patients may benefit from long-term pharmacologic treatment after surgery and follow-up with a multidisciplinary team including a pulmonologist, but currently we don't have a protocol for these patients.

Dr Thierry Mesana (Ottawa, Ontario, Canada). Congratulations for this large and interesting study. I noticed you had $12 \%$ mortality in those with high severe $\mathrm{PH}$, and you basically had almost no mortality in the patients with leaflet prolapse. Does this mean that most of your mortality was in the patients without organic disease, such as ischemic patients?

Dr Ghoreishi. The number of patients with severe $\mathrm{PH}$ was low in patients with degenerative MR. In the entire group, more than $30 \%$ of patients had moderate or severe $\mathrm{PH}$, and patients with higher degrees of preoperative $\mathrm{PH}$ were more likely to have functional and rheumatic MV disease.

Dr Mesana. Patients with severe PH may not have the same level of $\mathrm{PH}$ at the time of operation. So when did you take your measurements to consider the patient as having severe $\mathrm{PH}$ ? You can have a patient with a pulmonary systolic pressure of 60 before surgery, going down to 40 during anesthesia, and it is the same patient. So when did you qualify the patient with severe $\mathrm{PH}$ ?

Dr Ghoreishi. Determination of the presence and degree of $\mathrm{PH}$ in this study was based completely on preoperative evaluation, not intraoperative assessment.

Dr Chitwood. No Swan-Ganz?

Dr Ghoreishi. No.

Dr Mesana. No Swan-Ganz in the operating room?

Dr Ghoreishi. We routinely measure the pulmonary artery pressure intraoperatively, but the results in this study are based on catheterization-derived SPAP performed preoperatively.

Dr Chaw-Chi Chiu (Kaohsiung, Taiwan). You didn't mention the diastolic pulmonary artery pressure in your presentation. As we know, elevated diastolic pulmonary artery pressure is important to evaluate the reversibility of $\mathrm{PH}$ after the operation. 
Usually, if the differences between the diastolic pulmonary artery and the left ventricular end-diastolic pressures are more than 10 $\mathrm{mm} \mathrm{Hg}$, it would mean irreversibility of the PH even after the operation. I would like to suggest measuring the diastolic pulmonary pressure before operation to evaluate if the $\mathrm{PH}$ will decrease after operation.

Dr Dan Lindblom (Stockholm, Sweden). I am currently working in Africa on different humanitarian projects, mainly in Sudan on a big project where we are doing a lot of valve surgery. Last year, we performed more than $600 \mathrm{MV}$ procedures, obviously mainly for mitral stenosis in young patients. More than half of our patients came to surgery with a pulmonary pressure more than $60 \mathrm{~mm} \mathrm{Hg}$, and supersystemic pulmonary pressures were not uncommon.

In your experience, do you have any cutoff line where you would refuse the patient for surgery because of extreme $\mathrm{PH}$ ? What precautions would you take if you have a patient with very high pulmonary pressures?

Dr Ghoreishi. Twenty-five patients in this study had an sPAP greater than $80 \mathrm{~mm} \mathrm{Hg}$, and operative mortality was significantly higher (17\%) among those patients. However, we do not have any absolute cutoff point to refuse surgery because a good number of patients with significant $\mathrm{PH}$ can expect some symptomatic benefit from operation. 